Noname manuscript No.

(will be inserted by the editor)

\title{
The Dead-Alive Physicist Gedankenexperiment Debunked
}

\author{
Amal Pushp
}

Received: date / Accepted: date

\begin{abstract}
We refute a recently proposed thought experiment namely the Dead-Alive Physicist (DAP) whose authors claim it to be the falsification of the von Neumann interpretation in Quantum Mechanics. We discover major flaws in their model and our justification serves the purpose of disregarding further claims and assertions made in the paper at various stages. Thus, in a yet another situation, it is proven that the formalism advanced by von Neumann is non-trivial and non-falsifiable.
\end{abstract}

Keywords Quantum Superposition · Interpretations of Quantum Mechanics · State Vector Reduction · Measurement Problem · von Neumann Interpretation

\section{Introduction}

There had been various attempts to provide an explanation for the transition from a state of potentialities to a state of actuality in a quantum measurement. Each attempt, known as an interpretation, tries to address the problem from its own guiding principles [1-15]. Among these, two schools of thought exists between physicists and physics philosophers regarding the existence of a quantum state, one is ontic and the other is epistemic. An epistemic interpretation such as QBism can avoid the measurement problem totally, however, the Pusey-Barrett-Rudolph theorem rules out the possibility of such an interpretation $[16,17]$. Nevertheless, out of the so many quantum mechanical interpretations, one of the least supported and the one regarding which there are so many misconceptions is von Neumann's idea that the observer's consciousness is responsible for the collapse of the quantum wave function [18]. The Nobelist Eugene Wigner also supported this interpretation for a period of time [19]. Famous physicists in recent times who support this idea are Henry

Amal Pushp

Department of Physics, School of Basic Sciences, Manipal University Jaipur, Rajasthan303007 India E-mail: amal.201006004@muj.manipal.edu 
Stapp and Adrian Kent [20-23]. In [24] , the authors claimed to have empirically falsified the Consciousness Causes Collapse Hypothesis (CCCH) using delayed choice quantum eraser experiment [25]. However recently, researchers have strongly refuted their claims [26, 27, 28]. In [29], the basic ideas of $\mathrm{CCCH}$ has been employed to understand quantum paradoxes such as the measurement problem and the contrasting and conflicting behaviour of classical and quantum particles in a double-slit.

In this paper we focus our attention on another recent proposed experiment aimed at putting to rest the $\mathrm{CCCH}$ [30]. We show that the method employed by them is not a logical way to disprove in general any physical theory and in particular, the $\mathrm{CCCH}$.

The paper is organised in the following manner. In section 2 we provide a brief mathematical treatment of the process of state vector reduction and also see what von Neumann's idea has to say about it. Then in section 3 we introduce the DAP experiment and provide a logical refutation of the claims propounded by the same. The paper is closed with some important conclusions.

\section{Collapse of The Quantum State Vector}

Consider a quantum superposition state of the form,

$$
|\Psi\rangle=\sum_{\zeta}|\zeta\rangle\langle\zeta \mid \Psi\rangle
$$

It should be noted that the above state exists before any measurement has been made on the system. The above equation uses the identity operator $\hat{I}=$ $\sum_{\zeta}|\zeta\rangle\langle\zeta|$ in some fixed orthonormal basis $\{|1\rangle,|2\rangle, \ldots,|\zeta\rangle\}$ as follows:

$$
|\Psi\rangle=\hat{I}|\Psi\rangle=\sum_{\zeta}|\zeta\rangle\langle\zeta \mid \Psi\rangle=\sum_{\zeta}(\langle\zeta \mid \Psi\rangle)|\zeta\rangle=\sum_{\zeta} a_{\zeta}|\zeta\rangle
$$

where the quantum probability amplitudes have been set as, $a_{\zeta}=\langle\zeta \mid \Psi\rangle$. If we express the quantum state $|\Psi\rangle$ explicitly in the basis $\{|1\rangle,|2\rangle, \ldots,|\zeta\rangle\}$, it is obtained as,

$$
|\Psi\rangle=\left(\begin{array}{c}
a_{1} \\
a_{2} \\
\vdots \\
a_{\zeta}
\end{array}\right)
$$

Now the probability of each variable $\zeta$ being measured is given by the Born rule as,

$$
P(\zeta)=\langle\Psi|\hat{\mathbb{P}}| \Psi\rangle=\langle\Psi \mid \zeta\rangle\langle\zeta \mid \Psi\rangle=a_{\zeta}^{*} a_{\zeta}=\left|a_{\zeta}\right|^{2}
$$

An explicit computation of the same can be done using matrix multiplication,

$$
\left(\begin{array}{llll}
a_{1}^{*} & a_{2}^{*} & \ldots & a_{\zeta}^{*}
\end{array}\right)\left(\begin{array}{cccc}
0 & 0 & \ldots & 0 \\
0 & 0 & \ldots & 0 \\
0 & 0 & \ddots & \vdots \\
0 & 0 & \ldots & 1
\end{array}\right)\left(\begin{array}{c}
a_{1} \\
a_{2} \\
\vdots \\
a_{\zeta}
\end{array}\right)=a_{\zeta}^{*} a_{\zeta}=\left|a_{\zeta}\right|^{2}
$$


As soon as the measurement yields the outcome $\zeta$, the state vector reduces to a new state given by,

$$
|\Psi\rangle \rightarrow \frac{\hat{\mathbb{P}}_{\zeta}|\Psi\rangle}{\sqrt{\left\langle\Psi\left|\hat{\mathbb{P}}_{\zeta}\right| \Psi\right\rangle}}
$$

where the state has been normalized. In simple terms it means that after the collapse the state is with absolute probability (equal to 1 ) in the eigenstate $|\zeta\rangle$ of the projection operator $\hat{\mathbb{P}}_{\zeta}=|\zeta\rangle\langle\zeta|$ and belonging to the eigenspace $\mathbb{V}_{\zeta}$. Note that although the state in eq. (6) is normalized, it has an extra pure phase factor,

$$
|\Psi\rangle \rightarrow \frac{\hat{\mathbb{P}_{\zeta}}|\Psi\rangle}{\sqrt{\left\langle\Psi\left|\hat{\mathbb{P}_{\zeta}}\right| \Psi\right\rangle}}=\frac{1}{\left|a_{\zeta}\right|} \hat{\mathbb{P}}_{\zeta}|\Psi\rangle=\frac{1}{\left|a_{\zeta}\right|}\left(\begin{array}{c}
0 \\
0 \\
\vdots \\
a_{\zeta}
\end{array}\right)=\left(\begin{array}{c}
0 \\
0 \\
\vdots \\
e^{i \theta}
\end{array}\right)
$$

with the phase factor being computed using the knowledge of polar form of complex numbers viz. $a_{\zeta}=r e^{i \theta}$ where $r=\left|a_{\zeta}\right|$ and $\theta$ is the counter-clockwise angle from the real axis, in the following manner,

$$
\frac{a_{\zeta}}{\left|a_{\zeta}\right|}=\frac{r e^{i \theta}}{r}=e^{i \theta}
$$

Thus we have a well defined state vector before the measurement, $|\Psi\rangle$, and a well defined state vector after the measurement, $|\zeta\rangle$. Unambiguously, the quantum reduction of the state vector can be represented by $|\Psi\rangle \rightarrow|\zeta\rangle$ or $\sum_{\zeta} a_{\zeta}|\zeta\rangle \rightarrow|\zeta\rangle$. This is the essence of what happens when a quantum mechanical state vector collapses to a new state. Now from the perspective of the $\mathrm{CCCH}$, the collapse shouldn't take place unless a conscious observation is made. The above point is something which should be quite obvious. Each possible element of a superposition state is known beforehand and one can write down the mathematical form of the state which is nothing but the linear combination of the known elements in a particular basis. However, one never observes a superposition of any kind. Physically, the observer would find the system in a single definite eigenstate. The boundary that separates an unobservable superposition state and the observed eigenstate is a measurement which can be made either by a measuring device or a conscious observer or even by both in succession. The superposition would never actually get destroyed for an observer unless it is registered in his consciousness. This is also the solution to the measurement problem provided by this interpretation.

\section{The DAP Experiment}

We first discuss briefly the main points of the proposed experiment and then provide our response to the claims made in the paper. The setup involves a 
physicist inside a sealed room. On the ceiling a photon source L has been attached which would emit a single photon as and when required and is followed by a beam splitter BS, vertically in line with the photon source. Two photodetectors D and D' are fixed beyond the beam splitter and the efficiency of both of them is taken to be $100 \%$. D is located along the path of the photon and is fixed on the top of a box. D and D' have an angular separation of $90^{\circ}$. Inside the box there is a hammer and a glass flask containing a fatal gas called LGD. The emitted photon from L would be in a state of superposition after reaching the beam splitter BS, given by the following equation

$$
|\Psi\rangle_{\text {Photon }}=\left(\frac{|T\rangle+|R\rangle}{\sqrt{2}}\right)
$$

where the kets represent the transmitted and the reflected parts of the photon wave function. The experiment is supposed to be working in the following manner: Photon reaches detector $\mathrm{D} \rightarrow$ Hammer gets activated $\rightarrow$ Flask breaks down and gas is released $\rightarrow$ the physicist dies. If $\mathrm{D}$ gets activated, the physicist results into the state $|D e a d\rangle$ and if $\mathrm{D}^{\prime}$ gets activated the end result is the state $\mid$ Alive $\rangle$. The experiment has been formulated along the similar lines as that of the Schrodinger's cat scenario. Where the latter relies upon the radioactive decay of atom, the former depends on the probabilistic behavior of the photon. The crux of the matter in [30] is however the introduction of CPB strategy which distinguishes it from the Schrodinger's cat (and all concomitant experiments) and is also the prime reason which leads the authors to the faulty claim that their experiment falsifies the von Neumann postulation. This would be justified in a while from now.

The strategy requires that the physicist drugs himself with a Conscious Perceptions Breaker (CPB) so that he is not able to watch the experiment directly and is unconscious throughout the course of the experiment. The physicist comes back to his normal experience only at 1:00 PM. The state during the course of the experiment, i.e. between 12:00 - 1:00 PM is formulated in [30] as the entangled state,

$$
|\Psi\rangle_{\text {System }}=\left(\frac{\left.|T, D\rangle \otimes \mid \text { Dead }\rangle+\left|R, D^{\prime}\right\rangle \otimes \mid \text { Alive }\right\rangle}{\sqrt{2}}\right)
$$

Note that we have slightly modified the mathematical presentation of equations (9) and (10) from the original for clarity. At this moment there is entry of another person called W (supposedly playing the role of Wigner from the Wigner's Friend). It has been stated in [30] that W is a supporter of the CCCH and her intention is to open the room at 1:30 PM inorder to check whether $\mathrm{P}$ is dead or alive, while taking the necessary precautions by wearing a gas mask. Two possible outcomes have been listed by the authors as a result of this action which would be elaborated later on. In the subsection following this, we re-examine the claims of [30] and classify them as consistent and inconsistent. Moreover, we justify why they are inconsistent. 


\subsection{Accurate and Erroneous Conclusions of The DAP Analysis}

While reading the paper on the DAP experiment we discovered that some of the statements made by the authors are infact true and surprisingly, in support of the $\mathrm{CCCH}$ and on the other hand there were claims which were found to be quite inconsistent.

*The authors rightly point out that on the occasion that the CPB were not used, P's consciousness would cause the collapse of the wave function as soon as $\mathrm{P}$ has become aware of whether he was bound to die or to survive.

*The authors do accept the fact that in a quantum physics experiment the observer has to perform a necessary measurement/observation inorder to gain knowledge about the state of the system and that in this case the consciousness is transitive and plays an active role on the surrounding physical environment.

The above points seem to be accurate enough and coherent with the principles of the CCCH. However, the authors continue that since they have introduced the concept of $\mathrm{CPB}$ in their theory, the observer plays a passive role and the above facts doesn't fit the logic of the DAP experiment hence it falsifies CCCH. It is not arduous to infer from this that they are artificially and forcibly trying to disrupt the conditions on which a theory is based and then if the experiment doesn't yield the outcome that it is supposed to yield, they are claiming the theory itself doesn't work. For instance, if A is supposed to give an outcome $\mathrm{B}$ under a condition $\mathrm{C}$ and one tries to disturb the state of A by eliminating A's correlation with $\mathrm{C}$, the end result would not come as $\mathrm{B}$ in any chance. This is the blunder committed by the authors of [30]. Moving ahead, they state (which we are quoting exactly as in the paper),

"In fact $\mathrm{P}$, as soon as conscious at 1:00 PM, is neither in the same situation of the observer who deliberates to open the Schrödinger's box for verifying the state of the cat nor in the same status of attentiveness of Wigner's friend when checking whether he did perceive a flash or did not."

The above is quite apparent considering our justification of the previous point. How can the physicist be expected to be in the state as quoted above, if he was under the effect of CPB during the course of the empirical test? Another mistake committed by them is that they are trying to show the emergence of $\mathrm{P}$ 's consciousness as an effect of the wave function collapse. They assert that the appearance must have been the effect (rather than the cause) as a result of the wave function collapse supposed to have occurred long before when D' detected the photon. A little logic applied here would reveal that the appearance was infact due to the reason that the CPB reaction faded away!

Earlier we saw that two possible outcomes were listed in [30] as a result of $\mathrm{W}$ entering the framework. According to the first outcome $\mathrm{W}$ would find $\mathrm{P}$ in the state $|D e a d\rangle$ and would conjecture that it was her own act which caused the collapse of the wave function from the superposition state $|\Psi\rangle_{\text {Physicist }}=$ $\frac{\alpha \mid \text { Dead }\rangle+\beta \mid \text { Alive }\rangle}{\sqrt{2}}$ to the definite eigenstate $\mid$ Dead $\rangle$. On the other hand outcome 2 expresses that $\mathrm{W}$ would find $\mathrm{P}$ in the state $\mid$ Alive $\rangle$ and would enquire him 
about the experiment, to which P's response is that he is conscious from the past half hour. $\mathrm{W}$ then concludes that the wave function collapse occurred at sharp 1:00 PM, thus removing the dead state from the superposition but the authors write that W's conjecture is wrong because they believe that the emergence of P's consciousness and the collapse of the wave function are not distinct processes. Their belief is based on the assumption that at 1:00 PM only one event is taking place and that is, $\mathrm{P}$ realizing that he is alive. Also, according to them there would be no superposition of macroscopically distinct states $(\mid$ Dead $\rangle$ and $\mid$ Alive $\rangle)$ since the collapse has already taken place at the time D' received the photon. We discover a serious discrepancy in this claim. First of all we need to accept the fact that when two or more observers are involved in the same experiment entailing a superposition state, the collapse of the wave function would happen at different instances for different observers due to their independence from each other, unless there is some sort of communication between them. In this case, $\mathrm{W}$ and $\mathrm{P}$ lack any communication channel prior to their interaction. Taking these points into consideration, $\mathrm{W}$ is right when she states in outcome 1 that it was her own act of conscious observation which collapsed the wave function but exclusively only for herself! Similarly in outcome 2, the physicist didn't know whether the photon hit detector D or D' (because of the CPB effect) and hence for him there would be a superposition of the macroscopically distinct states discussed above, in some corner of his mind. As soon as it is 1:00 PM, the superposition state now reduces to the state $\mid$ Alive $\rangle$ for the physicist.

At this point, we have addressed more or less all the necessary claims and have shown the inconsistency of the vague and fallacious ones. In particular we discovered three major errors made in the formulation of the DAP thought experiment and provided the justification for the same. This directs us to the only cessation that the experimental model created by the authors of [30] fails to falsify the von Neumann premise. As a matter of fact we believe that the DAP proposal instead helps to validate von Neumann's interpretation as it bestows yet another testing ground for the theory.

If one really has to test this interpretation using the approach as in [30], the first thing that should be done is to abandon the CPB strategy, which is causing all the complications. It is not surprising that as soon as this is done, the experiment would now run smoothly in accordance with the von Neumann interpretation. For an augmentation one can then modify the DAP after resolving the issues we found and probe its Hilbert space setting along with the linear and non-linear evolution of the system, but we doubt that it would lead to any novel culmination different than those yielded by the analysis of [26]. Hence we don't feel it wise to repeat the same thing here.

\section{Concluding Remarks}

von Neumann's interpretation, just like any other formulation of quantum mechanics provides solution to the issues encircling the foundations of quan- 
tum theory. Although there have been instances where proposals were made claiming its falsification but to the contrary it has passed them all. There are two class of physicists who criticize this interpretation, first those who do not provide the basis on which they make the judgement and second those who actually come up with empirically testable model. For the former, we can attribute Stapp's thought on this on why the critics do so [20]. According to him its "metaphysical prejudice", in simple terms it means that there are metaphysical variables associated with the interpretation and generally physicists don't want to accept anything but physics. However, many other interpretations of quantum mechanics such as Everett's interpretation and de Broglie-Bohm theory involve such variables which are currently beyond the scope of testable mainstream physics (in particular the former requires the concept of parallel universes and the latter requires an infinite dimensional universe without a clear mechanism of how it leads to a three dimensional universe in our perception). Consequently, it is not wise to reject ideas simply because they are not yet testable or not yet in everybody's knowledge. Coming to the second class, the testable models they offer have suffered severe refutation on grounds that they contain several misconceptions and flaws, such as [24]. In this paper we explored another one of those [30]. In physics, we can't prove any theory. We can only verify it to a more accurate precision but we can nonetheless disprove a theory through repeated experimental tests. An empirical model can be refuted by finding logical incompatibility in its basics and we believe that [30] belongs to similar category. As we saw earlier in section 3 that [30] can't be considered as a disproof of $\mathrm{CCCH}$ because at first the authors don't really understand what disproof of a physical theory really means. A physical theory stands disproved if it doesn't work owing to the predictions that it is supposed to make, provided that the range of its conditions on which it is based is fulfilled. Rather than sticking to this what they really do is to introduce a factor which doesn't correlate with the principles of $\mathrm{CCCH}$ and is actually out of reach of the conditions of the theory, of course the theory will not work in this fashion, but this way it can't be considered a disproof but a mere misunderstanding. Anyway the authors should be commended for coming up with this thought experiment as it would only strengthen the von Neumann interpretation and prove its non-triviality and non-falsifiability yet again. Finally we believe that any theory in physics has its proponents and critics and similar is the case with $\mathrm{CCCH}$. However the important thing is that it should be considered in the same category as other interpretations of quantum mechanics, without being biased and which is also the main motive we have conveyed through this paper by debunking the DAP proposal.

\section{Acknowledgement}

The author would like to express gratitude to Dr. Danko Georgiev whose critical reading of the manuscript, explanations and suggestion of improvements in an earlier draft benefited him immensely in making the manuscript better. 


\section{References}

1. N. Bohr, The Quantum Postulate and the Recent Development of Atomic Theory, Nature. 121 580-590, (1928).

2. W. Heisenberg, Über den anschaulichen Inhalt der quantentheoretischen Kinematik und Mechanik, Z.Phys. 43 172-198, (1927). Translated by John Wheeler and Wojciech Zurek, in Quantum Theory and Measurement, (1983).

3. David Bohm, A Suggested Interpretation of the Quantum Theory in Terms of "Hidden Variables", I, Physical Review. (1952), 85, pp 166-179.

4. David Bohm, A Suggested Interpretation of the Quantum Theory in Terms of "Hidden Variables", II, Physical Review. (1952), 85, pp 180-193.

5. Hugh Everett, Relative State Formulation of Quantum Mechanics, Rev. Mod. Phys. 29(3) 454-462, (1957).

6. David Wallace, The Emergent Multiverse: Quantum Theory According to the Everett Interpretation. Oxford University Press (2012).

7. H. D. Zeh, "On the interpretation of measurement in quantum theory", Found. Phys. 1 (1) 69-76, (1970).

8. R. B. Griffiths, "Consistent histories and the interpretation of quantum mechanics", Journal of Statistical Physics 36 (1-2) 219-272, (1984).

9. John G. Cramer, "The Transactional Interpretation of Quantum Mechanics", Rev. Mod. Phys. 58 (3) 647-688, (1986).

10. Ruth E. Kastner, The Transactional Interpretation of Quantum Mechanics: The Reality of Possibility, Cambridge University Press (2012).

11. Ghirardi, G.C., Rimini, A., and Weber, T. Unified dynamics for microscopic and macroscopic systems, Phys. Rev. D. 34(2): 470-491, (1986). doi:10.1103/PhysRevD.34.470

12. Roger Penrose, On Gravity's role in Quantum State Reduction, Gen. Rel. Gravit. 28(5): 581-600, (1996). doi:10.1007/BF02105068

13. C. Rovelli, Relational Quantum Mechanics, International Journal of Theoretical Physics 35; 1996: 1637-1678. arXiv:quant-ph/9609002

14. Christopher A Fuchs, Rüdiger Schack. A Quantum-Bayesian Route to QuantumState Space Found. Phys. 41(3): 345-356, (2010). doi:10.1007/s10701-009-9404-8 https://arxiv.org/abs/0912.4252

15. Christopher A Fuchs, N. David Mermin, Rüdiger Schack. "An introduction to QBism with an application to the locality of quantum mechanics", American Journal of Physics. 82 (8) : 749-754, (2014). https://arxiv.org/abs/1311.5253

16. M. F. Pusey, J. Barrett, and T. Rudolph, On the reality of the quantum state Nature Phys. 8, 476 (2012). https://arxiv.org/abs/1111.3328

17. M. S. Leifer, Is the quantum state real? An extended review of $\psi$-ontology theorems Quanta 3, 67-155 (2014). https://arxiv.org/abs/1409.1570

18. John von Neumann, Mathematical Foundations of Quantum Mechanics, Princeton University Press (1955).

19. Eugene Wigner, Remarks on the Mind-Body Problem, in I. J. Good, ed.:The Scientist Speculates (Heinemann, London 1961, 284-302); Basic Books, New York (1962). Reprinted in E. Wigner: Symmetries and Reflections (Indiana University Press, Bloomington, Indiana 196, 171-184) and in J.A.Wheeler and W. H. Zurek eds.: Quantum Theory and Measurement (Princeton University Press 1983, 168-81)

20. H. Stapp, Quantum theory and the role of mind in nature, Found. Phys. 31, 1465-1499, (2001). arXiv:quant-ph/0103043

21. H. Stapp, Mind, Matter and Quantum Mechanics, (The Frontiers Collection) Springer (2009).

22. A. Kent, Quanta and Qualia, Found. Phys. 48, 1021-1037, (2018). arXiv:1608.04804 [quant-ph]

23. A. Kent, Collapse and Measures of Consciousness. arXiv:2009.13224 [quant-ph] (2020).

24. S. Yu, D. Nikolic, Quantum mechanics needs no consciousness Annalen der Physik 523(11), 931-938 (2011).

25. Kim, Y.H., Yu, R., Kulik, S.P., Shih, Y. and Scully, M.O. Delayed "choice" quantum eraser Phys. Rev. Lett. 84(1) 1-5 (2000). arXiv:quant-ph/9903047 
26. J.A. de Barros, G. Oas, Can we falsify the consciousness-causes-collapse hypothesis in quantum mechanics? Found. Phys. 47(10): 1294-1308 (2017). https://arxiv.org/abs/1609.00614

27. A. Knight, Quantum mechanics may need consciousness arXiv:2005.13317 [quant-ph] (2020).

28. C. M. Reason, arXiv:1707.01346 [quant-ph] (2017).

29. A. Pushp, S. Azmi, Proposal for solving the quantum measurement problem, PSTJ (Prespace. Jour.) 11(7): 592-600 (2020).

30. C. Roselli, B.R. Stella, The Dead-Alive Physicist experiment: a case-study disproving the hypothesis that consciousness causes the wave-function collapse in the quantum measurement process arXiv:2006.06368 [quant-ph] (2020). 